\title{
Clinical efficacy of betal selective adrenergic blockers in the treatment of neurocardiogenic syncope - a meta-analysis
}

This article was published in the following Dove Press journal:

Clinical Pharmacology:Advances and Applications

14 September 2010

Number of times this article has been viewed

\author{
Srikanth Vallurupalli ${ }^{\prime}$ \\ Smita Das ${ }^{2}$ \\ 'Division of General Internal \\ Medicine, Southern Illinois University \\ School of Medicine, Springfield, IL, \\ USA; ${ }^{2}$ Department of Kinesiology \\ and Community Health, University \\ of Illinois College of Medicine, \\ Champaign, IL, USA
}

Background: Beta1 $\left(\mathrm{B}_{1}\right)$ selective blockers have been widely used for the treatment of neurocardiogenic syncope though clinical trials have shown conflicting degrees of efficacy.

Objective: To study the clinical efficacy of $\mathrm{B}_{1}$ selective blockers compared to placebo in the treatment of neurocardiogenic syncope.

Methods: Four placebo controlled randomized studies were identified after search of existing English language literature. Review Manager (RevMan version 5, Oxford, England) was used for statistical calculations. Both random and fixed effects models were used for analysis.

Results: There was no demonstrable efficacy of $\mathrm{B}_{1}$ blockers compared to placebo even after a pre-specified sensitivity analysis. There was a trend towards more adverse events in the beta blocker group compared to placebo $(\mathrm{OR}=2.03 \mathrm{CI}=0.83-3.95, P=0.12)$.

Conclusion: There is no clinical evidence for justifying the use of $\mathrm{B}_{1}$ selective blockers in the treatment of adult neurocardiogenic syncope. These agents may in fact lead to a higher rate of adverse events compared to placebo.

Keywords: beta1 selective blockers, neurocardiogenic syncope

Neurocardiogenic syncope (or neurally mediated syncope) is a disorder of unknown pathogenesis which leads to transient loss of consciousness due to a failure of the body's reflex mechanisms to maintain adequate cerebral perfusion. ${ }^{1}$ It accounts for about $21.2 \%$ of all causes of syncope with an incidence of 6.2 per 1000 person years for first reported syncope. ${ }^{2}$ The exact pathogenesis of this disorder remains uncertain. ${ }^{3}$ There is no proven therapy though many classes of medications are prescribed. Both non selective and selective beta blockers have been used in clinical practice. There is an elevation of plasma catecholamines before both spontaneous and tilt-induced syncope, making beta adrenergic blockade an attractive option. They have also been postulated to decrease mechanoreceptor activation due to their negative inotropic effects thus preventing decreased venous return. ${ }^{4}$ Initial non randomized studies and one randomized controlled study added to the enthusiasm. However, recent randomized controlled studies have failed to prove the efficacy of selective $\beta 1\left(B_{1}\right)$ blockade in this disorder. We performed a meta-analysis to determine the clinical efficacy of oral $\mathrm{B}_{1}$ selective blockade in the treatment of neurocardiogenic syncope and to study the side effect profile compared to placebo.

\section{Methods}

70 I N First Street, PO Box 19636,

Springfield, IL 62794, USA

Tel + I 2175456914

Fax +I 2175457127

Email svallurupalli@siumed.edu

\section{Study identification}

We searched the PubMed, CINAHL, Web of Science, and Cochrane databases from inception to 2009 using MeSH terms "vasovagal syncope" "drug therapy" "adrenergic 
beta antagonists" and individually using the key words "vasovagal syncope" "neurocardiogenic syncope" "treatment" "metoprolol" " atenolol" "nebivolol" "bisoprolol" "acebutalol" "betaxolol" and "beta blocker". The "related article" feature was used to identify more related studies. References of individual articles were manually searched. HSRProj and National Research Register databases were used to identify any "grey" literature.

\section{Study selection}

The aim was to include randomized controlled studies that studied the clinical efficacy of oral $B_{1}$ blockade compared to placebo in patients above 18 years of age with a diagnosis of neurocardiogenic syncope. Since there is no therapy with proven efficacy in this disorder, we did not include studies that compared $\mathrm{B}_{1}$ blockers with another drug. Due to the absence of a "gold standard" for the diagnosis of this condition, a diagnosis of neurocardiogenic syncope as determined by the study investigators was considered acceptable as case definition. A careful cardiovascular and neurological investigation was considered essential inclusion criteria before such a diagnosis could be made. Clinical recurrence was defined as occurrence of syncope while on therapy or a lack of perceived benefit. Studies which reported only the effect on tilt-induced syncope were excluded. A total of hundred and sixteen studies were identified. Seven randomized controlled trials were identified through a process described in Figure 1. Four of these studies compared $\mathrm{B}_{1}$ blockade with placebo ${ }^{4-7}$ and one each with clonidine, ${ }^{8}$ propranolol, ${ }^{9}$ and no therapy. ${ }^{10}$ Only studies that included a placebo arm were included in the analysis. In the study by Brignole et al only the patients who underwent randomization were included in the analysis. ${ }^{4}$ Perceived lack of improvement was the outcome studied by Mahanonda et $\mathrm{al}^{6}$ while other studies reported rates of recurrence. Other than Madrid et al, ${ }^{5}$ studies only included patients who had a positive head up tilt table test as part of the case definition.

\section{Data extraction}

Two reviewers used a standard form to extract data independently. Disagreements were resolved after discussion. Efficacy data was extracted from the intention to treat analysis of all studies. The number of patients suffering adverse events was also identified.

\section{Quality of studies}

Each study was assigned a Jadad score by the two authors independently. A score of 3 or more was felt to be criteria for inclusion. After discussion, consensus was reached and the studies included were felt to be of satisfactory quality.

\section{Statistical analysis}

Review Manager (RevMan version 5, Oxford, England) was used for statistical calculations. Results of individual studies and overall result was expressed as an odds ratio (OR) with 95\% confidence intervals $(\mathrm{CI})$. A 2 -sided $P$ value less than 0.05 was considered statistically significant. $\mathrm{I}^{2}$ test was used as a measure of heterogeneity and the random effects model was used for analysis when significant heterogeneity (defined as $\mathrm{I}^{2}>50 \%$ ) was present. A post-hoc sensitivity analysis was planned in case of significant heterogeneity including studies with similar outcome measures and mean follow up duration.

\section{Description of studies included}

Table 1 describes the randomized controlled trials included in the meta analysis. Brignole and colleagues in 1992 randomized patients to drug therapy with various drugs including atenolol, dihydroergotamine, cafedrine, domperidone, and elastic compression stockings with or without drugs to placebo. ${ }^{4}$ Only the patients treated with atenolol alone $(n=7)$ were included and compared to the placebo group. The inclusion criteria required subjects to have two consecutive positive upright tilt table tests to be considered for the study. The study did not report a significant difference between any of the drug therapies compared to placebo. Atenolol was also compared to placebo in a randomized controlled study by Mahanonda et al. ${ }^{5}$ Patients with a history suggestive of vasovagal syncope were ruled out for structural heart disease and included in the study after a positive isoproterenol tilt table test. In addition to a statistically significant increase in patients reporting feeling better compared to placebo $(P=0.02)$, patients in the atenolol group had a drop in the number of episodes from $6 \pm 9.4$ /week to $0.6 \pm 1.6$ per week $(P=0.025)$. However, in the study by Madrid and colleagues, atenolol failed to decrease the recurrence of syncope compared to placebo. ${ }^{6}$ The median number of syncopal episodes during follow up was 2 in atenolol group and 0 in placebo group $(P=0.215)$. The largest trial and the only multicenter study to date was performed by Sheldon et al who randomized patients with a history of syncope and a positive tilt table test to metoprolol versus placebo. ${ }^{7}$ Metoprolol was no more efficacious than placebo in preventing recurrent syncope both in the intention to treat and on-treatment analysis. Sample size calculation with a study power of $80 \%$ was performed and reported in the latter three studies. ${ }^{5-7}$ 


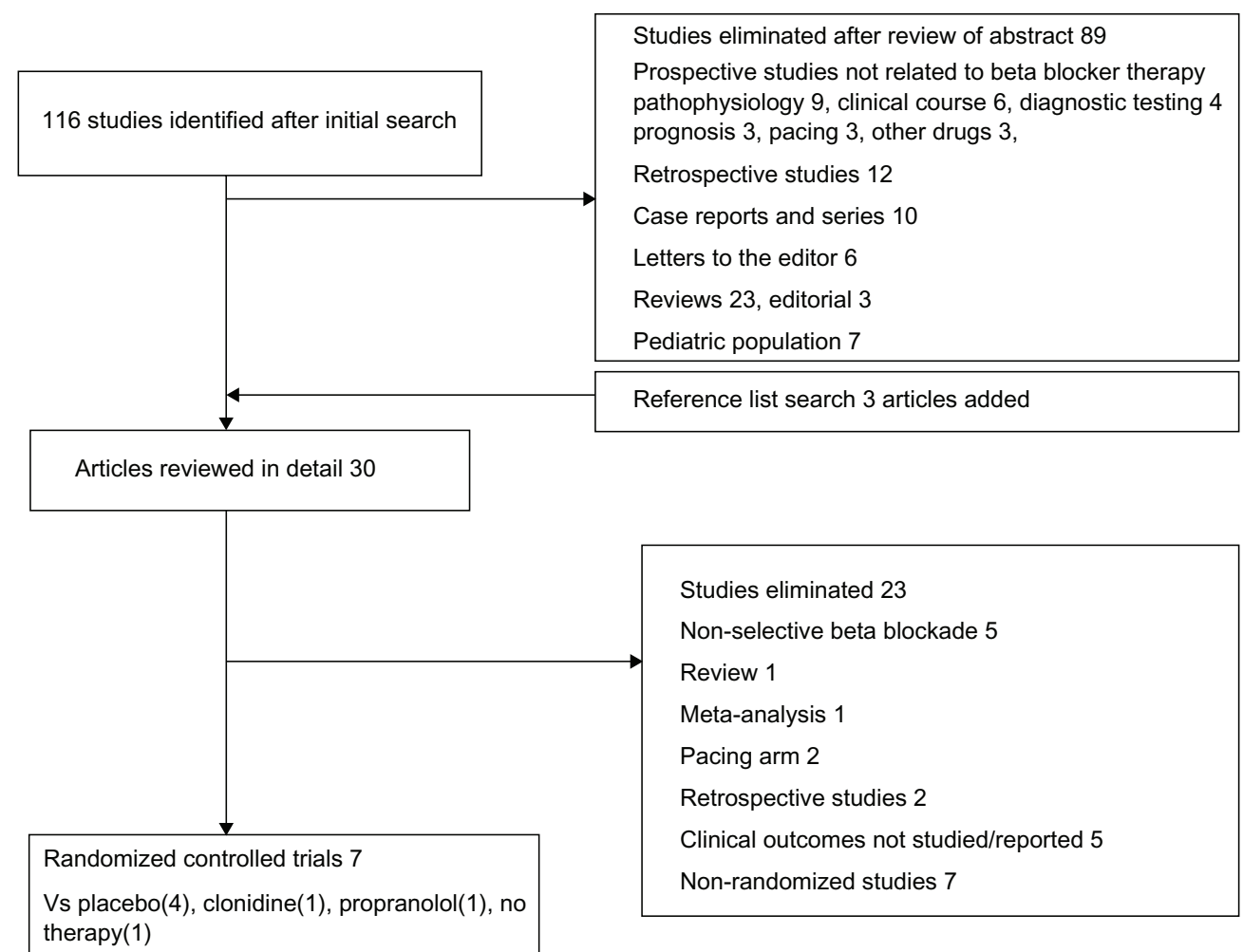

Figure I Flowchart describing the methods of study selection.

\section{Results of meta-analysis}

There was no demonstrable efficacy of $\mathrm{B}_{1}$ blockers compared to placebo as demonstrated in Figure 2. There was significant heterogeneity $\left(\mathrm{I}^{2}=64 \%\right)$ among the studies. When the analysis was repeated using a fixed effects model, there was still no statistically significant difference $(\mathrm{OR}=0.92, \mathrm{CI}=0.59-1.43$, $P=0.7)$. Using a pre-specified sensitivity analysis, the study by Mahanonda et al was excluded due to the shorter follow-up period ( 1 month), which yielded a $\mathrm{I}^{2}$ of $0 \%$ with an $\mathrm{OR}=1.18$ $(\mathrm{CI}=0.73-1.92, P=0.5)$. Further sensitivity analyses not specified before the study were completed in view of these results showing no efficacy. Analysis performed by excluding the study with the lowest number of subjects (Brignole et $\mathrm{al}^{4}$ which also did not report a sample size calculation) and by including only studies with atenolol (Brignole et al, Mahanonda et al and Madrid et al) did not reveal any significant benefit from using $\mathrm{B}_{1}$ blockers compared to placebo $(\mathrm{OR}=0.75$, $\mathrm{CI}=0.23-2.40, P=0.63$ and $\mathrm{OR}=0.69, \mathrm{CI}=0.13-3.59$, $P=0.66$ respectively). Significant heterogeneity persisted in both of these analyses ( $\mathrm{I}^{2}=74$ and $70 \%$ respectively). There were more adverse events in the beta blocker group (15 vs 8 , $\mathrm{OR}=2.03, \mathrm{CI}=0.83-3.95, P=0.12$ ). Funnel plot analysis was not performed due to the small number of studies since such an analysis may be misleading. ${ }^{11}$

\section{Discussion}

Neurocardiogenic syncope is a common disorder that results in transient loss of consciousness. The pathophysiology of this disorder is incompletely understood. Some have even questioned the existence of this disorder as a separate entity. In patients who are predisposed to this condition, reduced venous return from the lower extremities causes a decrease in the preload; the resultant decrease in cardiac output and blood pressure causes activation of the baroreceptor reflex. ${ }^{3}$ This causes an increase in ventricular contractility which is sensed by mechanoreceptors in the heart which project to the medullary dorsal vagal nucleus. A withdrawal of peripheral sympathetic tone and an increase in the vagal tone ensues resulting in vasodilation and bradycardia leading to clinical symptoms. This mechanism however discounts the importance of central mechanisms, which may contribute to syncope. There is some evidence of the importance of serotoninergic pathways which is supported by the evidence of the response of some patients to serotonin reuptake inhibitors. ${ }^{12}$ Several drugs including non selective and selective beta blockers, clonidine, midodrine, fludrocortisone, and SSRIs have been studied in the treatment of this disorder. ${ }^{5}$ Beta blockers (both selective and non selective) are commonly used medications in the treatment of patients diagnosed with neurocardiogenic 


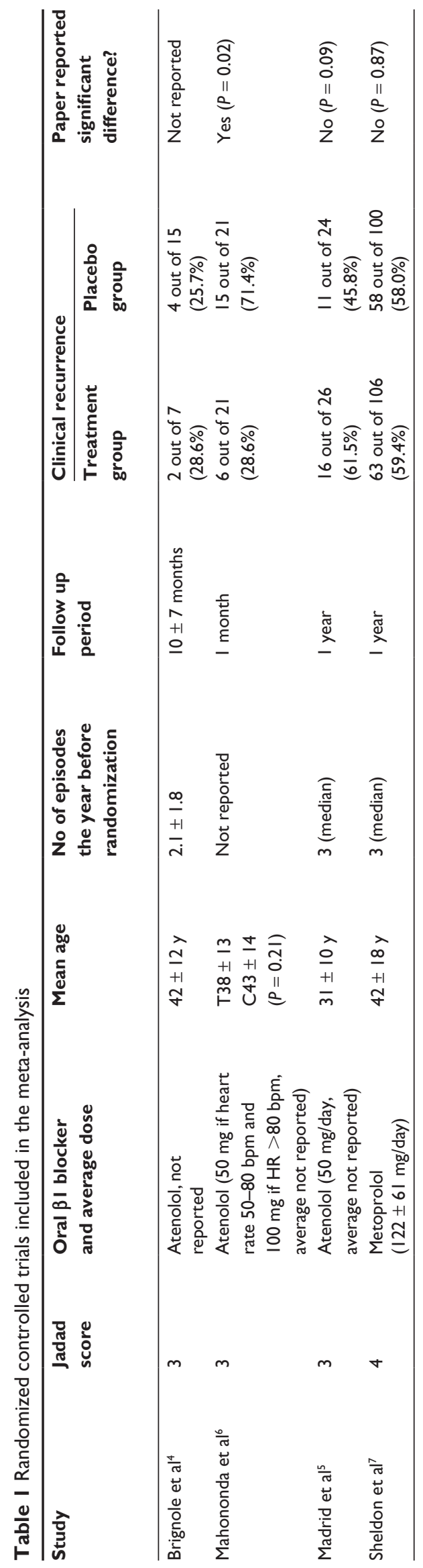

syncope. We chose to study the rates of clinical recurrence of syncope while on $B_{1}$ blocker therapy because of the practical clinical importance rather than study the response to tilt. We found no statistically significant improvement in clinical rates of recurrence of syncope with $\mathrm{B}_{1}$ blockers. There was actually an increased risk of adverse events though statistically not significant. The lack of clinical efficacy of $\mathrm{B}_{1}$ blockers may relate partly to the lack of understanding of the pathophysiology of neurocardiogenic syncope. The initial non randomized studies may simply have reflected the placebo nature of the administration of these medications. Adrenoreceptor stimulation in the heart may not play a major role in provoking syncope and thus selective $\mathrm{B}_{1}$ blockers may be ineffective. Non selective beta blockers such as propranolol may in fact be more effective by virtue of inhibition of the vasodilatory effect of activation of $\mathrm{B}_{2}$ receptor. ${ }^{13}$ In view of this difference; we did not include non selective blockers in this analysis as it would constitute an inhomogeneous treatment group. However, a randomized, placebo controlled study by Flevari et al failed to show the efficacy of non selective beta blockers (propranolol and nadolol) compared to placebo. ${ }^{14}$

Haghjoo et al reported similar efficacy of propranolol and metoprolol but the study lacked a placebo arm and the efficacy of these drugs may simply relate to a placebo effect. ${ }^{9}$ There may be a small subgroup of patients who may benefit from $B_{1}$ blocker therapy but this has not been adequately represented in these trials. Sheldon et al studied two such subgroups in a prespecified analysis based on age (less than or greater than 42 years) and need for isoproterenol to provoke a positive tilt study and neither group appeared to benefit from $B_{1}$ blocker therapy.

\section{Limitations}

We included only randomized controlled trials that included a placebo arm. There is evidence for a strong placebo effect in the treatment of neurocardiogenic syncope and the importance of including a placebo arm while studying this disorder has been described..$^{15}$ This prompted the exclusion of one randomized study (Ventura et al) which included a no treatment arm. ${ }^{10}$ This meta-analysis thus included only a small number of studies $(n=4)$. We also did not consider studies comparing $B_{1}$ blockers to other medications (for example, non-selective $\beta$-blockers) because the comparative drug may in fact be no better than placebo. We feel that these strict inclusion criteria actually enhance the value of this analysis. There was significant heterogeneity among the studies, which resolved with the deletion of one study in the pre specified sensitivity analysis. Heterogeneity persisted in the other sensitivity analysis, which failed 


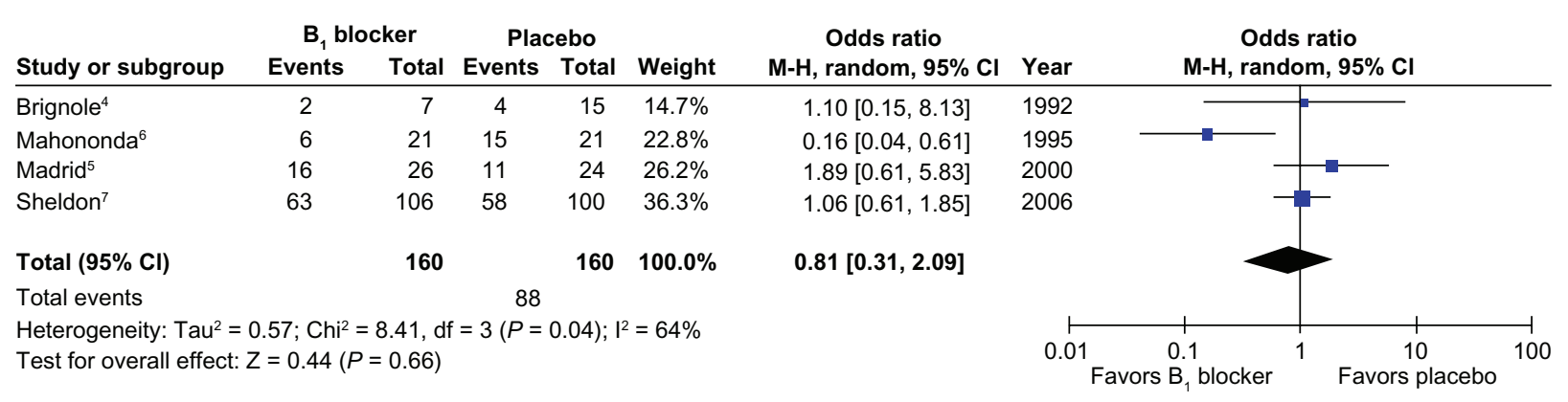

Figure 2 Forest plot illustrating the lack of clinical efficacy of $\mathrm{B}_{1}$ blockers compared to placebo.

to prove clinical efficacy. Atenolol is a hydrophilic $\mathrm{B}_{1}$ blocker while metoprolol by virtue of its lipophilic nature may cross the blood-brain barrier more readily. The treatment arm thus does not include medications with similar mechanisms of actions. However, studies with atenolol alone did not reach significance in the sensitivity analysis. Another limitation common to all studies of neurocardiogenic syncope is the variable course of the disease with some patients experiencing long periods of remission between episodes of syncope ${ }^{16}$ Some patients may even experience spontaneous remission. This complicates the assessment of the clinical efficacy of a drug in the treatment of this condition.

\section{Conclusion}

We performed this meta analysis to study the clinical efficacy of $\mathrm{B}_{1}$ adrenergic blockade in preventing clinical recurrence of syncope and to determine the rate of adverse events compared to placebo. Our results indicate a lack of clinical efficacy of these drugs and in fact a statistically non significant increase in adverse events. More randomized controlled studies are required to identify other effective therapies for this poorly understood disorder and a placebo arm should be a part of all such studies.

\section{Disclosure}

No conflicts of interest were declared in relation to this paper.

\section{References}

1. Tan MP, Parry SW. Vasovagal syncope in the older patient. J Am Coll Cardiol. 2008;51:599-606.

2. Soteriades ES, Evans JC, Larson MG, et al. Incidence and prognosis of syncope. N Engl J Med. 2002;347:878-885.
3. Alboni P, Brignole M, Degli Uberti EC. Is vasovagal syncope a disease? Europace. 2007;9:83-87.

4. Brignole M, Menozzi C, Gianfranchi L, Lolli G, Bottoni N, Oddone D. A controlled trial of acute and long-term medical therapy in tilt-induced neurally mediated syncope. Am J Cardiol. 1992;70:339-342.

5. Madrid AH, Ortega J, Rebollo JG, et al. Lack of efficacy of atenolol for the prevention of neurally mediated syncope in a highly symptomatic population: A prospective, double-blind, randomized and placebocontrolled study. J Am Coll Cardiol. 2001;37:554-559.

6. Mahanonda N, Bhuripanyo K, Kangkagate C, et al. Randomized double-blind, placebo-controlled trial of oral atenolol in patients with unexplained syncope and positive upright tilt table test results. Am Heart J. 1995;130:1250-1253.

7. Sheldon R, Connolly S, Rose S, et al. Prevention of syncope trial (POST): A randomized, placebo-controlled study of metoprolol in the prevention of vasovagal syncope. Circulation. 2006;113:1164-1170.

8. Biffi M, Boriani G, Sabbatani P, et al. Malignant vasovagal syncope: a randomised trial of metoprolol and clonidine. Heart. 1997;77:268-272.

9. Haghjoo M, Ali M, Fazelifar AF, Alizadeh A, Sadr-Ameli MA. A Randomized trial of oral propranolol versus metoprolol in treatment of neurally mediated syncope. Indian Heart J. 2006;58:426-431.

10. Ventura R, Maas R, Zeidler D, et al. A randomized and controlled pilot trial of beta-blockers for the treatment of recurrent syncope in patients with a positive or negative response to head-up tilt test. Pacing Clin Electrophysiol. 2002;25:816-821.

11. Lau J, Ioannidis JP, Terrin N, Schmid CH, Olkin I. The case of the misleading funnel plot. BMJ. 2006;333:597-600.

12. Grubb BP, Karas BJ. The potential role of serotonin in the pathogenesis of neurocardiogenic syncope and related autonomic disturbances. J Interv Card Electrophysiol. 1998;2:325-332.

13. Mathias CJ. Beta-adrenergic receptor blockers and the treatment of vasovagal syncope: more nails in the coffin! Clin Sci (Lond). 2006;111: 189-191.

14. Flevari P, Livanis EG, Theodorakis GN, Zarvalis E, Mesiskli T, Kremastinos DT. Vasovagal syncope: a prospective, randomized, crossover evaluation of the effect of propranolol, nadolol and placebo on syncope recurrence and patients' well-being. J Am Coll Cardiol. 2002;40:499-504.

15. Sheldon R, Rose S. Components of clinical trials for vasovagal syncope. Europace. 2001;3:233-240.

16. Barlow MA, Krahn AD. The role of pacing in neurocardiogenic syncope. Card Electrophysiol Rev. 1999;2:369-372.

\section{Dovepress}

\section{Publish your work in this journal}

Clinical Pharmacology: Advances and Applications is an international, peer-reviewed, open access journal publishing original research, reports, reviews and commentaries on all areas of drug experience in humans. The manuscript management system is completely online and includes a very quick and fair peer-review system, which is all easy to use.

Visit http://www.dovepress.com/testimonials.php to read real quotes from published authors. 